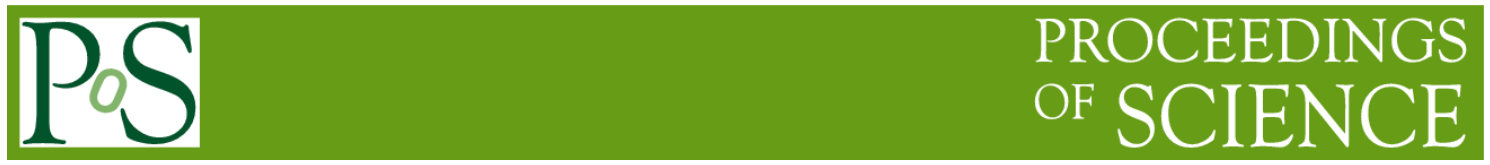

\title{
Silicon Devices Optimised for Avalanche Multiplication
}

\author{
Virginia Greco ${ }^{1}$ \\ IMB-CNM-CSIC \\ Campus Universidad Autónoma de Barcelona, 08193 Bellaterra \\ Barcelona, Spain \\ E-mail: Virginia.Greco@imb-cnm.csic.es
}

P. Fernández-Martínez; D. Flores; S. Hidalgo; G. Pellegrini; D. Quirion; M. Baselga IMB-CNM-CSIC

Barcelona, Spain

\section{N. Cartiglia}

INFN

Torino, Italy

\section{G. Kramberger}

University of Ljubljana

Ljubljana, Slovenia

\section{S. Grinstein}

IFAE/ICREA

Barcelona, Spain

J. Lange

IFAE

Barcelona, Spain

M. Fernández-García; I. Vila

IFCA (CSIC-UC)

Santander, Spain

C. Gallrapp; M. Moll

CERN

Geneva, Switzerland

V. Fadeyev; H. Sadrozinski SCIPP

Santa Cruz, US

R. Mori; U. Parzefall

University of Freiburg

1

Speaker 
Freiburg, Germany

\title{
R. Bates
}

Glasgow University

Glasgow, UK

G. Casse

University of Liverpool

Liverpool, $U K$

\begin{abstract}
We give an overview about new silicon devices for particle detectors that exploit the avalanche phenomenon of a reverse-biased pn junction to give an internal moderate gain. These new devices, the Low Gain Avalanche Detectors (LGAD), offer proportional response and good efficiency as the PiN diodes, and also a higher signal, while the noise in not increased significantly. In this paper we present the results of an extensive characterization campaign.

The LGAD diodes -developed and fabricated at IMB-CNM (Barcelona, Spain)- exhibit good electrical characteristics (current stability and high breakdown) before and after irradiation. Studies carried out with red laser TCT have shown that both front and back surface (before irradiation) are very uniform. The devices also offer excellent performances before irradiation in terms of charge collection, with a gain of $\sim 10$. Nevertheless, a severe decrease of the gain has been observed after neutron or proton irradiation at high fluences, so that further studies are ongoing in order to increase their radiation resistance.

Timing measurements have also shown that the LGAD detectors are ideal candidates for the realization of silicon detectors with very good space and time resolution, for 4-dimensional tracking of charged particles. Such devices could be applied as time-of-flight detectors for physics experiments, and as particle counters for dosimetry.
\end{abstract}

The 23rd International Workshop on Vertex Detectors

15-19 September 2014

Macha Lake, The Czech Republic 


\section{Introduction}

Silicon detectors are largely employed in particle and nuclear physics as particle trackers because of the unique properties of silicon, which make it very suitable for the detection of ionizing radiation [1].

If operated in the linear region, reverse-biased p-on-n (PiN) diodes give a response that is proportional to the amount of charge generated by the primary ionization in the semiconductor. In addition, they offer good efficiency and large energy spectrum detection. This is because silicon is characterized by a small band gap, and the average energy needed for creating an electron-hole pair is only $3.6 \mathrm{eV}$. Therefore, a large number of charge carriers is produced for a given energy loss of the ionizing particle.

On the other hand, PiN detectors' performance is negatively affected by thermal and electronic noise, and extended exposition to high irradiation fluences causes worsening of their signal to noise ratio.

A way to improve the performance of these devices is to make them generate a stronger signal while keeping the noise low. Such effect is produced in avalanche diodes (normally applied in optical physics), which are able to provide internal gain thanks to the mechanism of charge multiplication for avalanche [2]. They generate a signal much larger than the one produced by the primary ionization. Thanks to this internal gain, a lower gain electronic amplifier can be used: this will result in less electronic noise.

A new type of silicon particle detector has been developed at the IMB-CNM-CSIC ${ }^{2}$ (Barcelona, Spain), within the CERN ${ }^{3}$ RD50 Collaboration [3], which uses the avalanche multiplication mechanism in order to obtain a controlled moderate gain and low noise.

\section{Diodes with internal gain}

The Low Gain Avalanche Detectors (LGAD) [4] are planar silicon detectors that offer the same good properties of PiN diodes together with an improved signal to noise ratio, due to the charge multiplication mechanism typical of avalanche diodes. They are characterized by a gain in the order of 10, which is low compared to the gain needed for application in other fields, and are meant to be used in their linear region.

The LGAD technology has been developed at first on the simplified geometry of a pad diode; in a second phase, its application to segmented detectors (strips and pixels) has been investigated: this study is still ongoing.

In this article we focus on LGAD diodes and we present an overview of measurements performed on these detectors in order to evaluate their performance before and after irradiation.

\section{LGAD structure}

The LGAD diodes are p-type pads in which a p-type (multiplication) layer is diffused below the $\mathrm{n}+$ electrode, so that an $\mathrm{n}+\mathrm{p} / \mathrm{p}-/ \mathrm{p}+$ junction is created along the centre of the device (Fig. 1). If the diode is polarized in reverse mode, a high electric field is generated in this localized region,

\footnotetext{
${ }^{2}$ Instituto de Microelectrónica de Barcelona, Centro Nacional de Microelectrónica, Campus Universidad Autónoma de Barcelona, 08193 Bellaterra - Barcelona (Spain).

${ }^{3}$ European Organization for Nuclear Research (CERN), Meyrin-Geneva (Switzerland).
} 
which can lead to avalanche multiplication of the electrons reaching the $n+$ electrode. The gain of the detector critically depends on the doping profile of the multiplication p-layer, whose purpose is to enhance the value of the electric field in the central area.

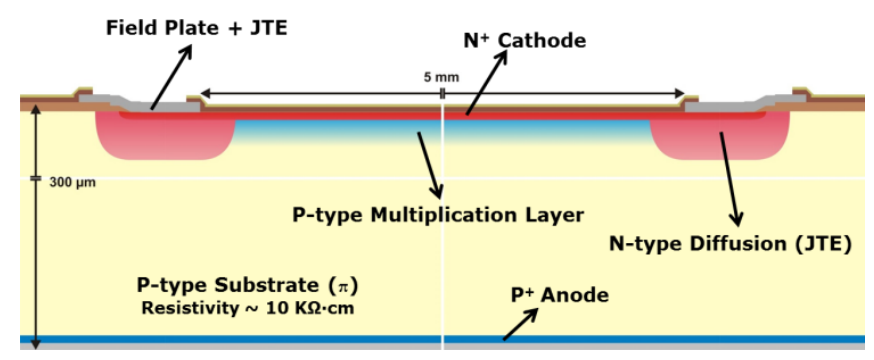

Fig. 1 Schematic cross-section of the LGAD diode design. A p-type multiplication layer is diffused below the $n+$ well of the top electrode. Special junction termination extensions (JTE) are included in order to reduce the electric field peak at the junction between the $n+$ cathode and the p-type multiplication layer.

A special Junction Termination Extension (JTE) has been designed in order to avoid early breakdown at the $\mathrm{n}+$ cathode/ $\mathrm{p}$-type multiplication layer junction, where the electric field shows a peak due to the border curvature and the high doping dose. It consists in overlapping the main junction edge with a n-type diffusion. Thanks to the JTE, the electric field peak in this area is reduced, so that the breakdown voltage can be lower than the $\mathrm{V}_{\mathrm{BD}}$ of the central junction. More details on the structure of the LGAD diode are given in [4].

The development of the LGAD technology has been carried out in the clean room facilities of IMB-CNM along different fabrication runs. In this article we report on the measurements performed on the detectors of the latest two runs ${ }^{4}$. The main difference between these two runs is that in one case a p-spray implantation was realized on the substrate; this is expected to isolate the active area and the guard ring. A design optimization of the LGAD main parameters was previously performed using the Sentaurus TCAD simulation toolkit [5].

Three different implant doses have been used for the p-type multiplication layer (with implantation energy of $100 \mathrm{keV}$ ):

- Dose A: $1.6 \times 10^{13}$ ions $\times \mathrm{cm}^{-2}$;

- Dose B: $2.0 \times 10^{13}$ ions $\mathrm{x} \mathrm{cm}^{-2}$;

- Dose C: $2.2 \times 10^{13}$ ions $\mathrm{x} \mathrm{cm}^{-2}$.

All other fabrication steps within the same run are equal, so changes in the behaviour shown by detectors from different wafers are to be ascribed to the different implant doses of the multiplication layer.

The LGAD diodes have an area of $5 \times 5 \mathrm{~mm}^{2}$ and are fabricated on high resistivity FZ substrates, $300 \mu \mathrm{m}$ thick. The physical junction sits at a depth of $1 \mu \mathrm{m}$ and the multiplication layer extends down to about $6 \mu \mathrm{m}$, as predicted by technological simulations and confirmed by inverse engineering technique analysis. On the front they have a (round or square) window opened in

\footnotetext{
${ }^{4}$ Namely: Run6474; and Run7062
} 
the metallization, while on the back there is an aluminium grid: both design solutions have been adopted in order to allow measurements with laser light.

The prototypes have been characterized to study their behaviour under typical working conditions. Electrical characteristics and charge collection performances have been tested at the IMB-CNM facilities before irradiation. Laser TCT measurements have also been carried out at CERN in order to study the signal generation in the bulk and the detector response uniformity. Some LGAD diode samples have been irradiated with neutrons or protons and their charge collection performances tested at IMB-CNM and, more extensively, at JSI ${ }^{5}$ in Ljubljana (Slovenia).Timing measurements have also been performed at CERN on un-irradiated LGAD diodes to evaluate their time resolution.

\section{Electrical characterization}

Leakage current and bulk capacitance as functions of the reverse applied voltages have been measured with a probe station for each device individually, before and after wafer dicing, in a controlled temperature and humidity environment $\left(\mathrm{T}=22^{\circ} \mathrm{C}, \mathrm{H}=70 \%\right)$.

The current-voltage curves of the LGAD diodes show that, once the full depletion is reached, the leakage current is in general stable up to $1100 \mathrm{~V}$ (upper limit of the range of measurement). Sensors with a higher implantation dose have larger leakage current, which is consistent with larger internal gain. Differences in the electrical characteristics are observed between detectors of the two different runs, as well as between devices of the same run (Fig. 2).
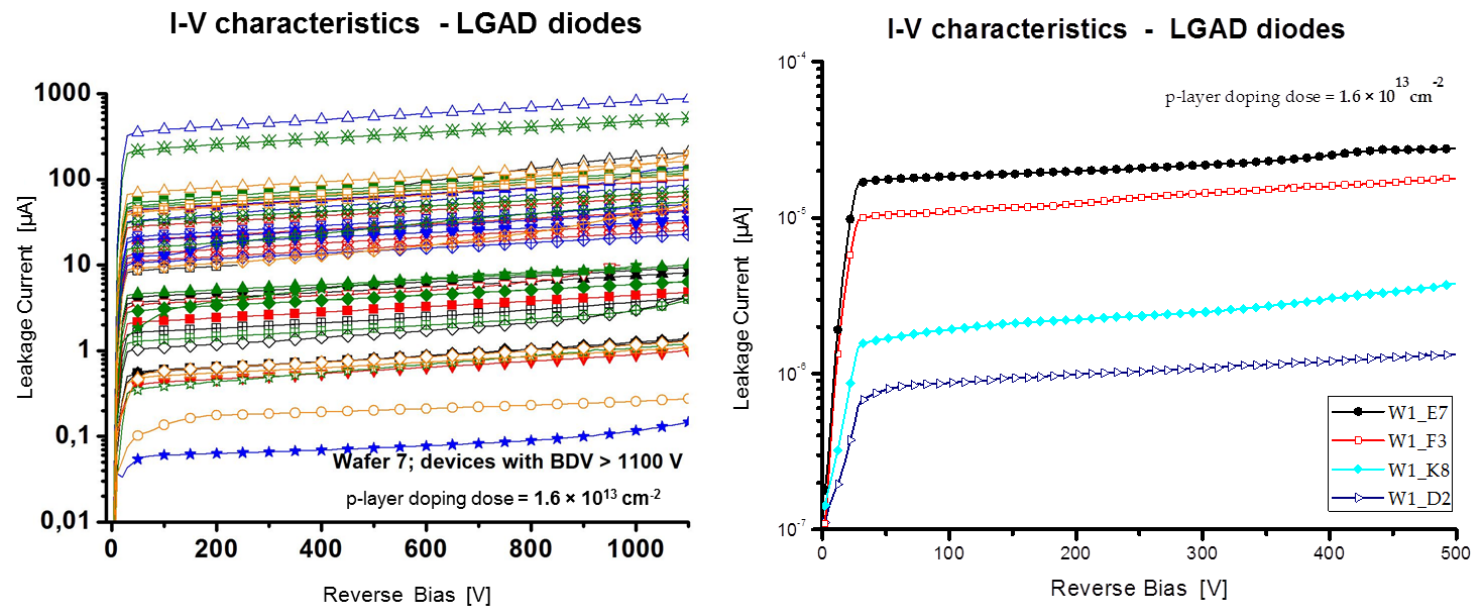

Fig. 2 I-V characteristics of LGAD diodes with doping dose $1.6 \times 10^{13} \mathrm{~cm}^{-2}$ (from two different runs). Once the full depletion is reached (around 40V), the current is generally stable (though slightly increasing). Differences in the electrical characteristics are observed between detectors of the two different runs, as well as between devices of the same run.

In Fig. 3 the maps of two wafers are shown: both have implantation dose A of the multiplication layer but the one on the left has p-spray. Each box represents one LGAD diode and the number in it is the intensity of the current corresponding to the voltage value indicated on the top $(300 \mathrm{~V})$. The colour varies on a continuous scale (given at the bottom) according to the current value. These maps show that the current-voltage characteristic is not homogeneous over the

\footnotetext{
${ }^{5}$ Jožef Stefan Institute, Ljubljana (Slovenia).
} 
wafer and that, on average, the devices of the latest run (wafer on the right in Fig. 3) have higher leakage currents.
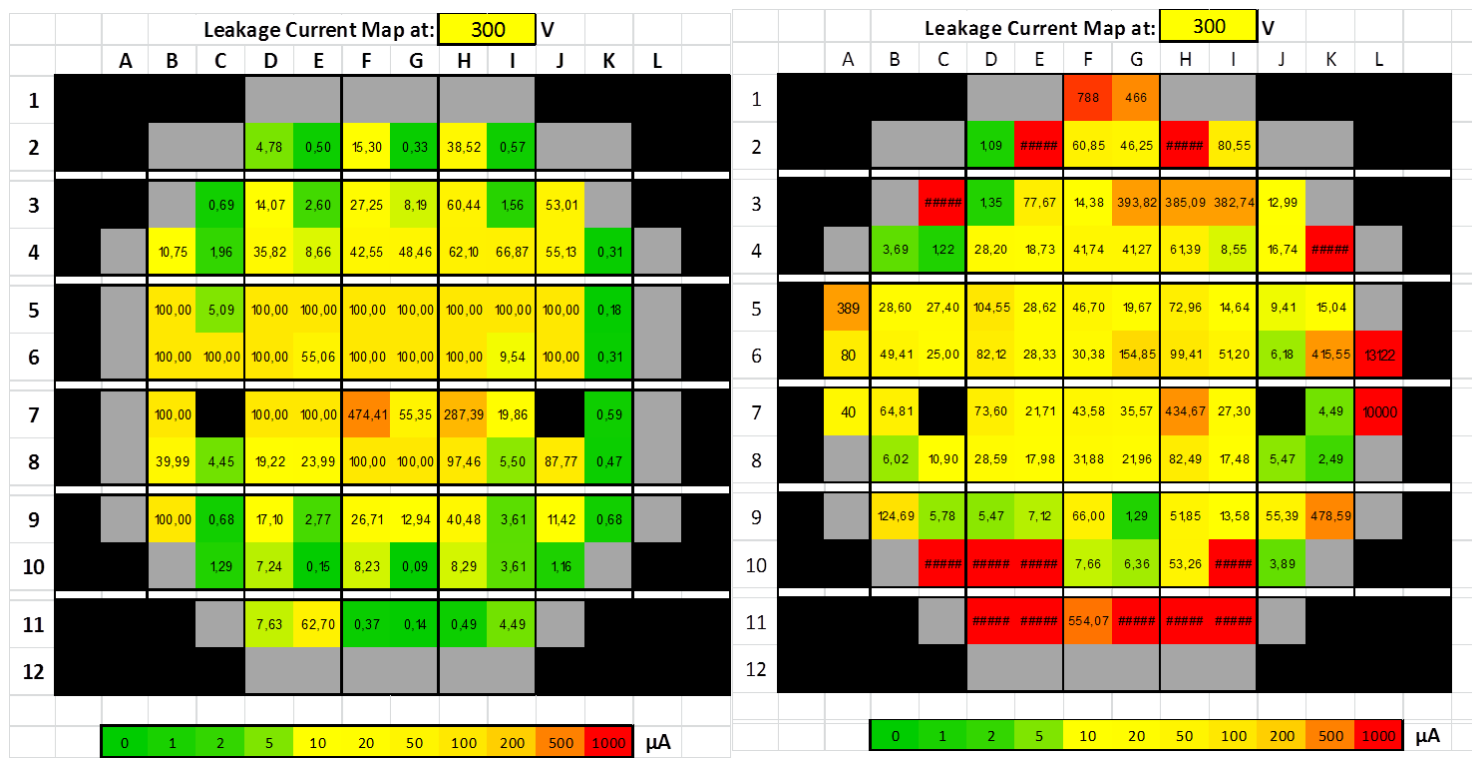

Fig. 3 Map of the wafers having implant dose 1,6x10 ${ }^{13} \mathrm{~cm}^{-2}$ : with p-spray (left, Run6474)) and without p-spray (right, Run7062). Each box represents one LGAD diode and the number in it is the intensity of its current (in $\mu \mathrm{m}$ ) at $300 \mathrm{~V}$. The color varies on a continuous scale, indicated at the bottom. "\#\#" means: current >0,1A.

A similar behaviour is observed in the diodes with implantation dose $\mathrm{B}$, which also present even higher currents. The diodes with dose $\mathrm{C}$ show a very steep current and reach the instrumentation compliance at relatively low voltage (between $60 \mathrm{~V}$ and100V).

In order to investigate the origin of the high current measured in the LGAD diodes of the latest run (without p-spray, Run7062), we studied its dependence on temperature (in the range [-40; $20]^{\circ} \mathrm{C}$ with $10^{\circ} \mathrm{C}$ steps). We observed little decrease of the measured current when reducing the temperature: this behaviour suggests that its major component is surface current [6]. The absence of the p-spray allows negative charges to accumulate on the surface and such inversion layer may facilitate the injection of surface currents in the diode active area. Further studies are being carried out in order to understand the cause of this increment of the leakage current as well as of its dispersion throughout the same wafer.

From the measurement of the capacitance as a function of the applied voltage, we can extract the full depletion voltage by using the method of the intercept [7]. The $\mathrm{V}_{\mathrm{FD}}$ values of the detectors are: $\sim 40 \mathrm{~V}$ for the devices with dose A of the multiplication layer, and $\sim 50-60 \mathrm{~V}$ for the devices with dose $\mathrm{B}$.

In Fig. 4 the inverse squared capacitance (as a function of the applied voltage) of a LGAD sample having dose $\mathrm{A}$ is plotted. It is possible to distinguish two knee points in the curve: $\mathrm{V}_{\mathrm{MR}} \sim 25 \mathrm{~V}$, and $\mathrm{V}_{\mathrm{FD}} \sim 40 \mathrm{~V}$. For $\mathrm{V}<25 \mathrm{~V}$ the value of $1 / \mathrm{C}^{2}$ is very low because the $\mathrm{p}$-type multiplication layer is not yet depleted; for bias voltages higher than $\mathrm{V}_{\mathrm{MR}}(\mathrm{MR}=$ multiplication region), the depletion of the bulk starts and the full depletion is reached around $40 \mathrm{~V}$. After that, $1 / \mathrm{C}^{2}$ keeps increasing linearly with $\mathrm{V}$ because of lateral depletion, up to $\sim 220 \mathrm{~V}$, where it 
becomes constant. $\mathrm{V}_{\mathrm{MR}}$ depends on the value of the effective doping dose of the multiplication layer [8].

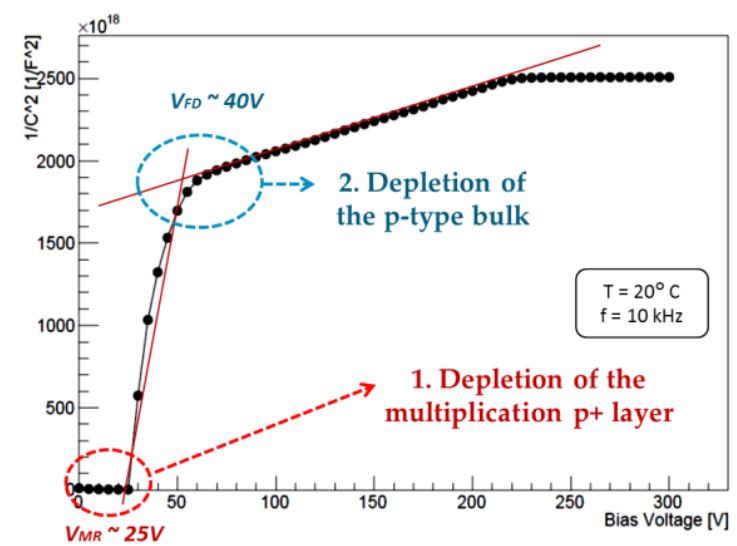

Fig. $41 / C^{2}$ curve of one LGAD sample with dose of the multiplication layer $1,6 \times 10^{13} \mathrm{~cm}^{-2}$.

\section{Laser TCT Measurements}

We performed Transient Current Technique (TCT) measurements [9] with red laser on LGAD diodes in order to verify the uniformity of the detector response, as well as to observe the signal generation inside the bulk.

In TCT measurements of LGAD diodes the transient current comprises both the free charge carrier currents generated directly by the laser in the primary ionization; and the signal due to charge multiplication (which is expected to be stronger). As a consequence, if we illuminate the reverse-biased LGAD diode from the back we measure a signal made up of:

- A current induced by the collection of electrons generated in the primary ionization, that are produced far from the cathode of the detector;

- A minor component of hole current due to the primary ionization;

- A current of holes generated by charge multiplication in the p-layer (which is set close to the top) and recollected by the bottom electrode;

The first two components sum up in a common peak, so they are indistinguishable one from the other, while we can clearly observe the hole current due to multiplication because it is generated with a delay.

Laser TCT measurements on LGAD samples have been performed at CERN (at the Detector Technology Group) by using a dedicated setup [10]. The equipment comprises: a picosecondpulsed red laser (wavelength 660nm); optics for front and back illumination; a stage able to move in three directions ( $\mathrm{x}, \mathrm{y}, \mathrm{z}$ coordinates) in micrometric steps; power supplies; a LabView based software to control data acquisition and loop parameters for complete scans. Voltage scans have been realised and the transient current collected. 
In Fig. 5 the plot of a voltage scan with back illumination, performed on a LGAD diode with dose $\mathrm{A}$ of the multiplication layer, is shown. The signal curves corresponding to low reverse bias voltages present a first peak mainly due to the current induced by electrons (which drift fast and are collected in less than 10ns), and a following plateau caused by the holes collection (which is slower because of the smaller mobility of holes). By integrating the charge signal over a $25 \mathrm{~ns}$ time interval, we obtain the curve of the collected charge as a function of the reverse bias voltage (see Fig. 6).

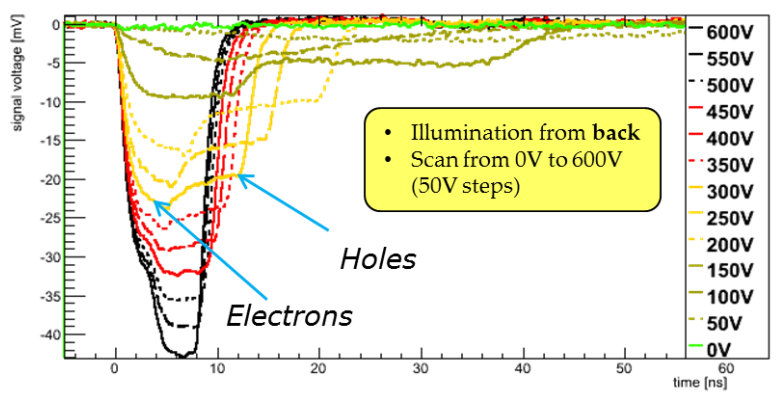

Fig. 5 Red laser voltage scan with back illumination of an LGAD diode (dose A of the multiplication layer). In the signal curves corresponding to low reverse bias voltages, two transient current components are clearly visible.

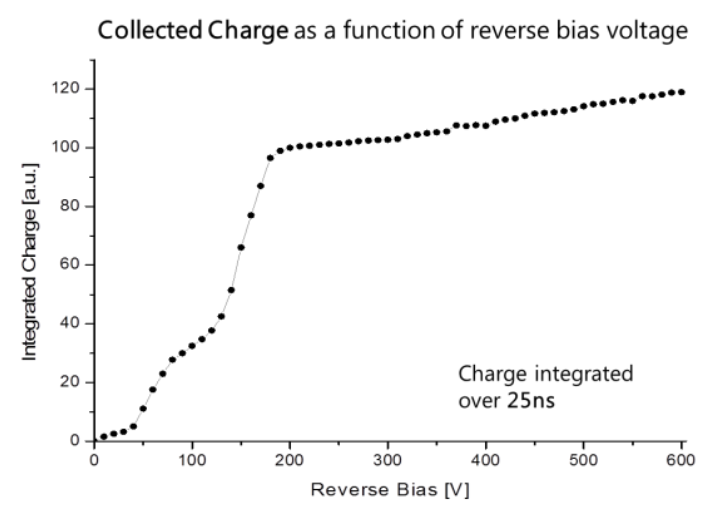

Fig. 6 Charge collected in the LGAD and integrated over a 25ns interval, as a function of the reverse bias voltage. The LGAD diode is illuminated from the back by red laser light.

A surface scan has also been carried out by illuminating the diode from the back side (through a circular hole in the PCB on which the detector is mounted) and from the front side, at different reverse bias voltages. Fig. 7-left shows the front side scan of a LGAD diode, having doping dose $\mathrm{A}$, done with $100 \mu \mathrm{m}$ steps at $150 \mathrm{~V}$. In Fig. 7-right the back side scan of the same diode, realized with $75 \mu \mathrm{m}$ steps at $150 \mathrm{~V}$, is represented. Both surfaces appear very homogeneous (the grid visible on the back surface is the ground metal grid of the detector), which means that the response of the diode is uniform. 

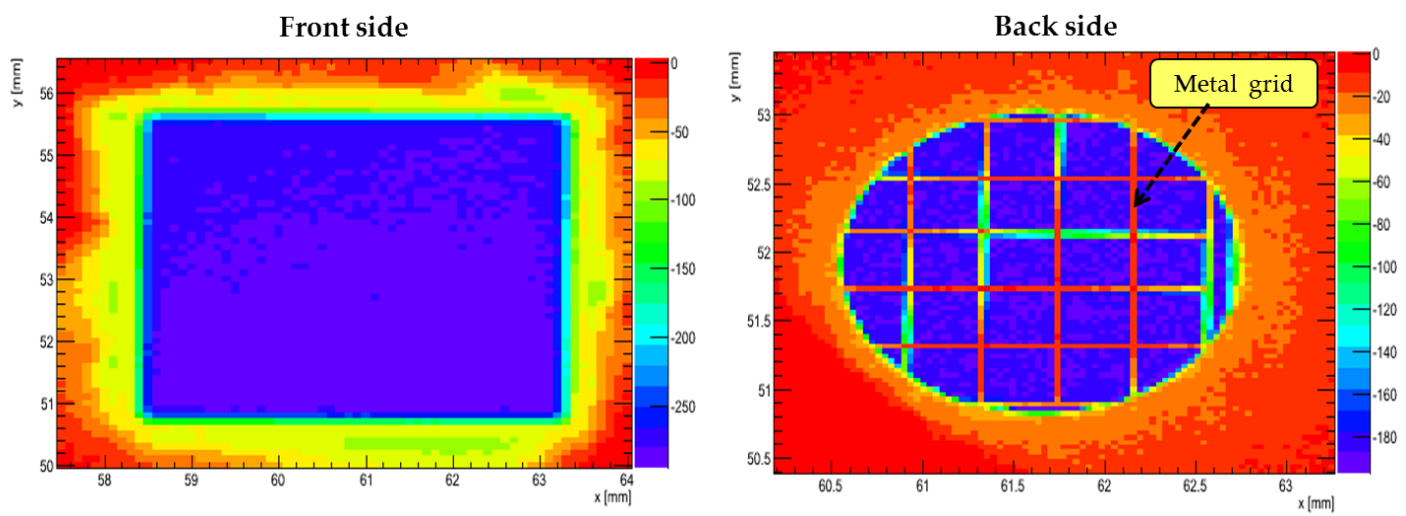

Fig. 7 Red laser surface scan of a LGAD diode with implant dose of the multiplication layer $1,6 \times 10^{13} \mathrm{~cm}^{-2}$, at $150 \mathrm{~V}$. Left: front side scan with 100

\section{Charge collection measurements}

Charge collection performances of the LGAD prototypes have been evaluated by exposing the detectors to a tri-alpha $\left({ }^{239} \mathrm{Pu} /{ }^{241} \mathrm{Am} /{ }^{244} \mathrm{Cm}\right)$ radiation source. The signal generated by the charge was treated by an electronic system made up of: a full custom readout board (specifically designed at IMB-CNM), a multi-channel analyser, and its software for collecting and visualizing data. The detector was illuminated from the back, where the surface is more uniform, and the reverse bias voltage gradually increased (from 10V to $1100 \mathrm{~V}$ ).

The charge collected in the LGAD prototypes is almost the same as in PiN diodes up to the full depletion voltage. But when increasing the voltage, the charge collected in the PiN's do not change, while in the LGAD diodes we observe an increment of the signal: no plateau is reached up to $1100 \mathrm{~V}$. It shows that a multiplication phenomenon is actually occurring. By comparing the value of the charge collected in the LGAD device with that in the PiN diode we obtain the gain at each value of the applied voltage [11].

Charge collection measurements have also been performed with minimum ionizing particles (MIP's), by irradiating the samples with a ${ }^{90} \mathrm{Sr}$ radiation source, at the JSI facilities in Ljubljana (Slovenia). A dedicated electronic system is used in order to read-out and to treat the signal, as well as a software package to analyse the data [8].

Both types of measurements show that on average the LGAD diodes having doping dose A offer a gain in the range [2-4], while those having dose B offer a gain of $\sim 10$ and in some case can reach a gain of 20 (Fig. 8). 

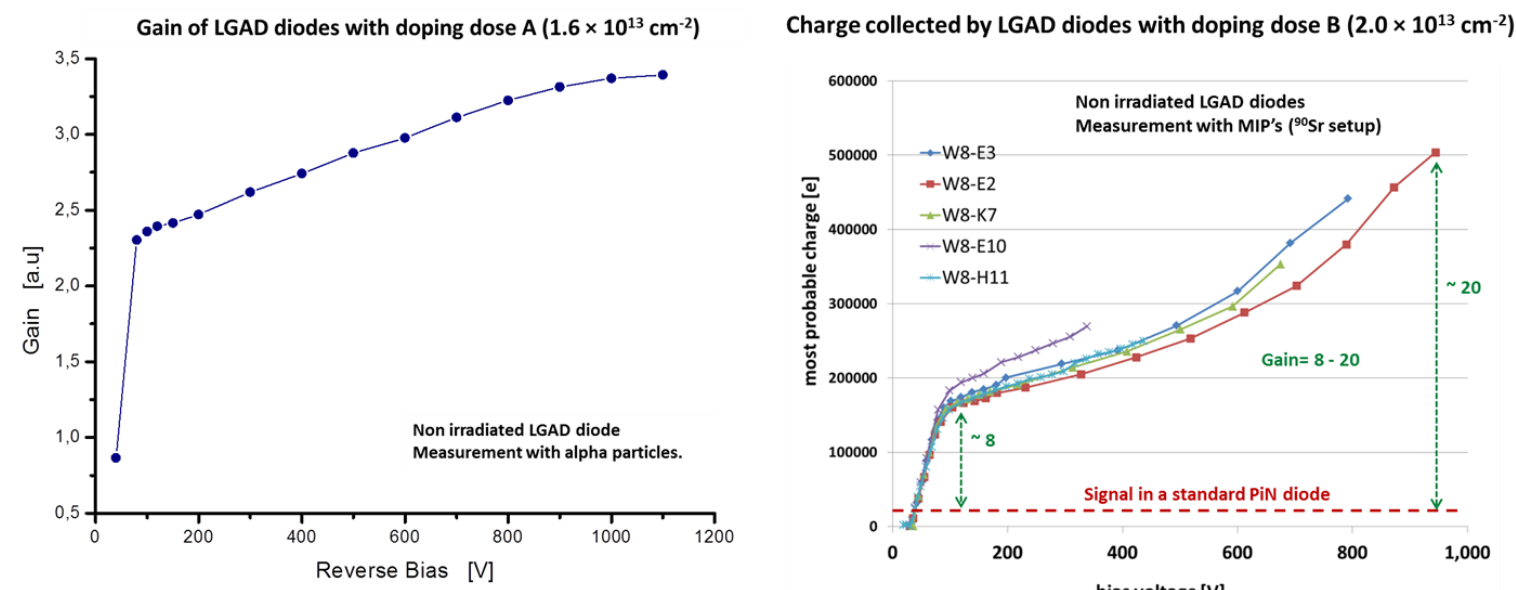

Fig. 8 (Left) Average gain of non-irradiated LGAD diodes with doping dose A $\left(1.6 \times 10^{13} \mathrm{~cm}^{-2}\right)$; the measurements were performed using an alpha particles source. (Right) Charge collected by non-irradiated LGAD diodes having doping dose $B\left(1.6 \times 10^{13} \mathrm{~cm}^{-2}\right)$; the measurements were performed using $a^{90}$ Sr source (i.e. a minimum ionizing particles (MIP's) source).

\section{Radiation effect studies}

A group of LGAD diodes with implant doses A and B of the multiplication layer were irradiated (in Ljubljana, Slovenia) in fractions with reactor neutrons, in order to evaluate the effect of irradiation at increasing fluences. The samples were annealed at $60^{\circ} \mathrm{C}$ for 80 minutes after each irradiation step and then the gain extracted from ${ }^{90} \mathrm{Sr}$ radiation measurements (as described in the previous section). The fluence steps were the following: $1 \times 10^{14} \mathrm{neq} \mathrm{cm}^{-2}, 2 \times 10^{14} \mathrm{neq} \mathrm{cm}^{-2}$, $3 \times 10^{14}$ neq $\mathrm{cm}^{-2}, 5 \times 10^{14}$ neq $\mathrm{cm}^{-2}, 1 \times 10^{15} \mathrm{neq}^{-2}, 2 \times 10^{15}$ neq $\mathrm{cm}^{-2}, 3 \times 10^{15}$ neq $\mathrm{cm}^{-2}$ (and $1 \times 10^{16}$ neq $\mathrm{cm}^{-2}$ for the diodes with doping dose B only).
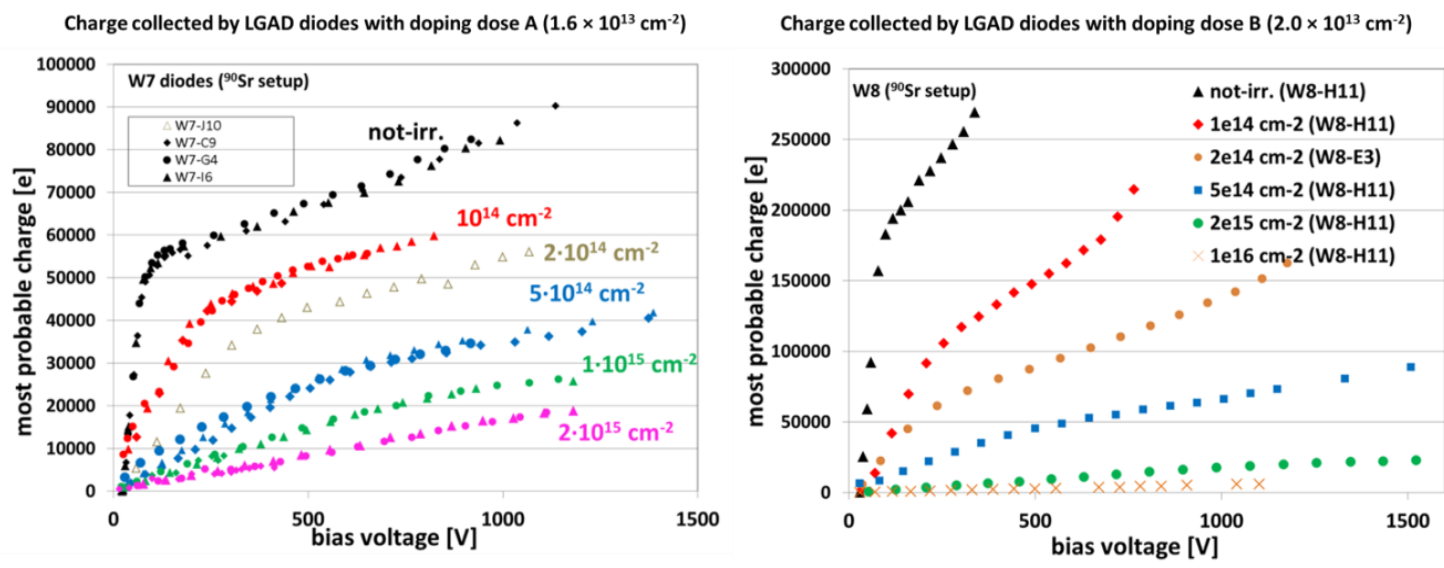

Fig. 9 Charge collection measurements (with MIP's) of LGAD diodes after neutron irradiation. Left: LGAD diodes with doping dose $1,6 \times 10^{13} \mathrm{~cm}^{-2}$; right: LGAD diodes with doping dose $2.0 \times 10^{13} \mathrm{~cm}^{-2}$.

In Fig. 9 the charge collected (most probable value) as a function of the bias voltage is plotted for LGAD diodes with doping dose A (left) and doping dose B (right) respectively. Such measurements show that the charge multiplication decreases with increasing irradiation fluence. 
Another group of LGAD diodes was irradiated at the Los Alamos National Laboratory in Los Alamos (US) with $800 \mathrm{MeV}$ protons. Different fluences were delivered, comprised between $1.4 \times 10^{13}$ neq $\mathrm{cm}^{-2}$ and $1 \times 10^{16}$ neq $\mathrm{cm}^{-2}$. The charge collection performance of the proton irradiated diodes have been evaluated, on some samples, with alpha particle measurements (after annealing) and, on the rest of the samples, with ${ }^{90} \mathrm{Sr}$ radiation measurements (without intentional annealing).

In both cases, we observed a reduction of the gain with increasing fluences. The gain of $800 \mathrm{MeV}$ proton irradiated LGAD diodes with implant dose A, measured with alpha particles, is plotted in Fig. 10-left. It is calculated by comparing the charge collected by irradiated LGAD diodes to the charge collected by standard PiN diodes. Fig. 10-right shows the charge collected by LGAD diodes with implant dose B (full symbols) and PiN diodes (open symbols) illuminated by a ${ }^{90} \mathrm{Sr}$ source (MIP's). From this plot, it is evident that heavy irradiation strongly affects both LGAD and PiN diodes and that the gain is almost irrelevant already at a fluence value of $9.9 \times 10^{13} \mathrm{neq}^{-2}$. This gain degradation appears to be attributable to removal of initial shallow acceptors and trapping (at high irradiation fluences) [8].
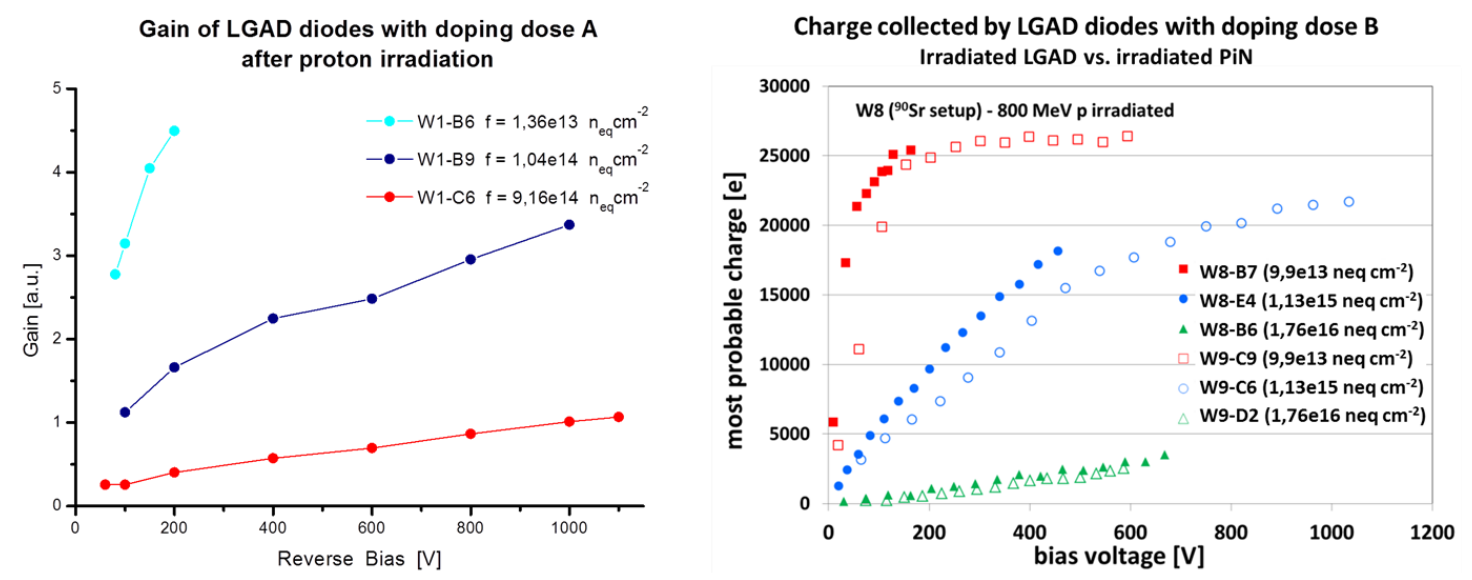

Fig. 10 Charge collection measurements of LGAD (and PiN) samples after 800MeV proton irradiation. Left: gain of diodes with doping dose $1.6 \times 10^{13} \mathrm{~cm}^{-2}$; measurements performed using an alpha particles source. Right: charge collected by diodes with doping dose $2.0 \times 10^{13} \mathrm{~cm}^{-2}$ (full symbols) and PiN diodes (open symbols); measurements performed using $a^{90} \mathrm{Sr}$ source.

\section{Time Measurements}

The charge multiplication phenomenon of LGAD devices can be exploited in order to obtain silicon detectors for 4-dimensional tracking of charged particles with very good space and time resolution $\left(\sigma_{\mathrm{t}}<50 \mathrm{ps}, \sigma_{\mathrm{s}} \in[20,50] \mu \mathrm{m}\right)$. Silicon pixel detectors have already sufficient position resolution, but normally determine time quite poorly. LGAD detectors could solve this problem and open the way to the realization of ultra-fast-silicon-detectors (UFSD) [12].

The time resolution $\sigma_{t}$ can be expressed as the sum of three terms: time walk, jitter, and TDC binning. Each of these components can be parameterized by using a simple model approximation, from which we infer that $\sigma_{t}$ depends on the rate of change of the output voltage 
per unit of time, which is the slew rate $\left(\frac{d V}{d t}\right)$. As a conclusion, in order to minimize the time resolution, we need to maximize the slew rate, which means that a large and short output signal is required. Simulations performed with a simplified model of diode detectors show that the slew rate is inversely proportional to the thickness of the device, the size being equal. Hence, in order to improve the time resolution it is necessary to fabricate thin detectors (bulk thickness $<100 \mu \mathrm{m})$.

The technology of LGAD devices appears to be particularly indicated for developing ultra-fastsilicon detectors, because of the increased $\mathrm{S} / \mathrm{N}$ and because of the higher amount of charge produced in the detector that would generate a sufficiently high signal even in a thin bulk.

First timing measurements have been performed at CERN by RD50 Collaboration members from INFN Torino ${ }^{6}$. Two LGAD pad diodes were illuminated with a split laser signal (wavelength $1064 \mathrm{~nm}$ ) and the time difference between the two signals was measured by a SAMPIC (Sampler for Picosecond Time Measurements) [13]. On the base of this time difference it is possible to estimate the jitter. The measurement was repeated with two standard PiN diodes and the results compared. As we can see in Fig. 11, the time jitter with LGAD diodes is much smaller: in particular, it goes down to 40ps while it is 100ps with PiN sensors. A strong reduction of the jitter translates into a high improvement of the time resolution.

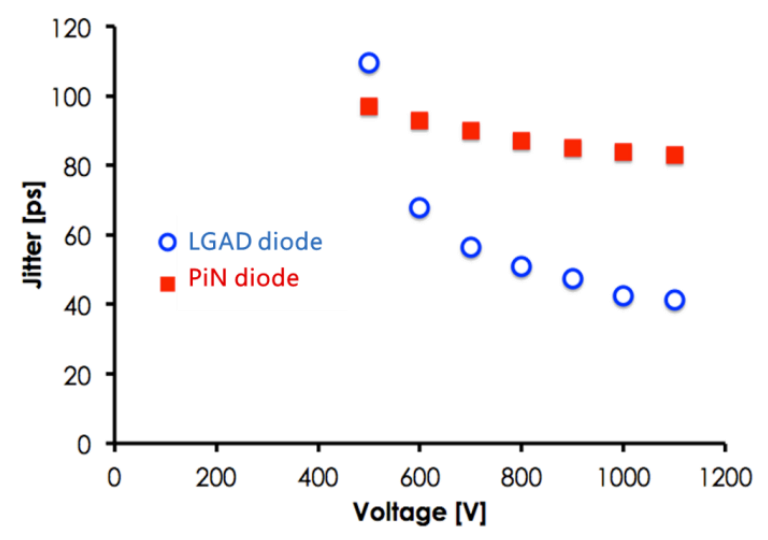

Fig. 11 Time jitter measurements performed with standard PiN diodes (red squares) and LGAD diodes (blue circles). The jitter with LGAD diodes is much smaller than with PiN diodes.

\section{Conclusions}

The avalanche phenomenon of a pn junction polarized in reverse mode has been exploited in order to develop new detectors with internal moderate gain, called Low Gain Avalanche Detectors (LGAD).

Prototypes have been fabricated at the CNM clean room facilities, with three different doping doses of the p-type multiplication layer. The electric characterization has shown good performances (current stability and high breakdown) for the LGAD diodes before and after irradiation. Detectors from the latest run, without p-spray, exhibit high leakage currents. Red

\footnotetext{
6 Istituto Nazionale di Fisica Nucleare (INFN), Torino (Italy).
} 
laser TCT voltage scans with front and back illumination (before irradiation) have shown that the detector response is uniform.

According to the results of charge collection measurements, the LGAD diodes exhibit excellent performances before irradiation: the multiplication phenomenon produces a gain of $\sim 3$ in LGAD diodes with implant dose of $1.6 \times 10^{13} \mathrm{~cm}^{-2}$ and a gain of $\sim 10$ (in some case even 20 at high voltages) in LGAD devices with implant dose of $2.0 \times 10^{13} \mathrm{~cm}^{-2}$.

Nevertheless, a severe decrease of the gain has been observed after neutron or proton irradiation at high fluences (up to $1 \times 10^{16}$ neq $\mathrm{cm}^{-2}$ ). This phenomenon is particularly evident in the case of proton $(800 \mathrm{MeV})$. Further studies and developments of the LGAD detectors will be carried out in order to increase their radiation resistance, such as investigating the possibility to use heavier dopants for the p-type multiplication layer implantation (such as gallium).

Timing measurements performed with laser light have shown that the LGAD detectors are ideal candidates for the realization of ultra-fast-silicon-detectors, which would combine the notorious excellent space resolution of silicon detectors to a highly improved time resolution, obtained by thinning the substrate of the sensor. A new fabrication run of LGAD detectors on thinner substrate is already in progress.

Thanks to their higher signal to noise ratio and to the possibility to improve their time resolution, the LGAD detectors are promising devices to be used for tracking purposes (in no harsh radiation environments, such as in the International Linear Collider), as well as in timing applications, such as: time-of-flight detectors for physics experiments, and particle counters for dosimetry.

\section{Acknowledgements}

The work presented in this article has been performed within the framework of CERN RD50 Collaboration. This research has been partially financed by the Spanish Ministry of Economy and Competitiveness (MINECO) through the Particle Physics National Program, grants: FPA2013-48308-C2-1-P; FPA2013-48387-C6-2-P; and SEV-2012-0234 (Severo Ochoa Excellence Program). The authors also acknowledge the support of the JAE-Doc Program of the Spanish Superior Council for Scientific Research (CSIC). The work at SCIPP was supported by the US Department of Energy, grant DE-FG02-13ER41983.

\section{References}

[1] G. Lutz, Semiconductor Radiation Detectors, Device Physics; Berlin, Heidelberg, New York; Springer, 2007.

[2] I. Tapan et al., Avalanche Photodiodes as Proportional Particle Detectors, Nuclear Instruments and Methods in Physics Research, vol. A388, pp. 79-90, 1997.

[3] CERN, RD50 Collaboration, http://rd50.web.cern.ch/rd50/. 
[4] G. Pellegrini, P. Fernández-Martínez, M. Baselga, C. Fleta, D. Flores, V. Greco, S. Hidalgo, I. Mandic, G. Kramberger, D. Quirion and M. Ullan, Technology developments and first measurements of Low Gain Avalanche Detectors (LGAD) for high energy physics applications, Nuclear Instruments and Methods in Physics Research, vol. A765, pp. 12-16, 2014.

[5] Sentaurus Device: an advanced multidimensional (1D/2D/3D) device simulator, Synopsys, http://www.synopsys.com/Tools/TCAD/DeviceSimulation/Pages/SentaurusDevice.aspx.

[6] A. Chilingarov, Temperature dependence of the current generated in Si bulk, Journal of instrumentation, vol. 8, P10003, 2013.

[7] A. Chilingarov, Recommendations towards a standardisation of the macroscopic parameter measurements, CERN - RD50 Collaboration, Geneva (Switzerland), 2003.

[8] G. Kramberger, Radiation hardness of Low Gain Amplification Detectors (LGAD), in 24th RD50 Workshop, Bucharest (Romania), 2014.

[9] V. Eremina, N. Strokana, E. Verbitskayaa and Z. Li, Development of transient current and charge techniques for the measurement of effective net concentration of ionized charges (Neff) in the space charge region of p-n junction detectors, Nuclear Instruments and Methods in Physics Research, A372, Issue 3, pp. 388-398, 01/04/1996.

[10] C. Gallrapp et al., TCT, eTCT and I-DLTS measurement setups at the CERN SSD Lab, in 24th RD50 Workshop, Bucharest (Romania), 2014.

[11] V. Greco et al., Preliminary Results of Measurements of Proton Irradiated LGAD Pad Detectors, 24th RD50 Workshop, Bucharest (Romania), 2014.

[12] N. Cartiglia et al, Performance of ultra-fast silicon detectors, Journal of instrumentation, vol. 9, C02001, 2014.

[13] E. Delagnes, D. Breton, F. Lugiez, R. Rahmanifard, The SAMPIC chip for timing detectors, Nucl. Science IEEE Transactions, Vol. 54, Issue 5, Part 2, p. 1735, 2007. 